\title{
End of the year issue: is a magic pill on its way? We can always dream
}

\author{
Giorgina Barbara Piccoli ${ }^{1}$
}

Published online: 16 November 2021

(c) Italian Society of Nephrology 2021

Now, as the troubled year 2021 is about to end, the rhythm of life is changing once again. Back to normal? No, not yet; in fact our idea of normality has probably changed in the past 2 years, while the pandemic ravaged our countries.

A few days ago The Washington Post announced the arrival of two wonder pills: "On Thursday, a different front opened up in the battle against COVID-19-one that was notable for revolving around pills, rather than jabs. Regulators in Britain granted approval to the experimental drug Molnupiravir from U.S. pharmaceutical giant Merck, marking the first time the promising pill-based treatment for COVID-19 had been authorized anywhere in the world" [1].

Meanwhile, a company based in Israel announced the start of clinical trials for its orally delivered vaccine candidate, which uses specially designed capsules, and a second company received funding for developing an oral vaccine tablet, while the World Health Organization announced that four other orally delivered and eight intranasal vaccine candidates are undergoing clinical trials [1].

Medicine has run at a different speed in recent years and the pandemic further accelerated the development of new technologies, as well as research on old and new drugs. In a moment when attention is focused on vaccine hesitancy, protests against the influence of the pharmaceutical companies, economic crises and social turbulence, we risk losing sight of the unprecedented medical advances that we are witnessing.

Are they heralds of a wonder pill capable of retarding dialysis or establishing life-long tolerance of kidney transplants?

We can always dream.

This particularly rich end of the year issue offers insights into the present state of the art in nephrology, and its multifaceted interests.

Giorgina Barbara Piccoli

gbpiccoli@yahoo.it

1 Nephrologie, Centre Hospitalier Le Mans, Avenue

Roubillard 186, 7200 Le Mans, France
It not only contains several original studies on large series of patients; but is also particularly rich in images and experiences that offer clinical nephrologists valuable lessons. The success of these new features is evidence of the curiosity and attention to intriguing cases and individual patients that continue to characterize our profession.

This is a message of hope, and with this message, let me wish that all of you-friends, editors, authors, reviewers and, above all, readers-will keep on loving our profession and dreaming of ways to improve it (Fig. 1).

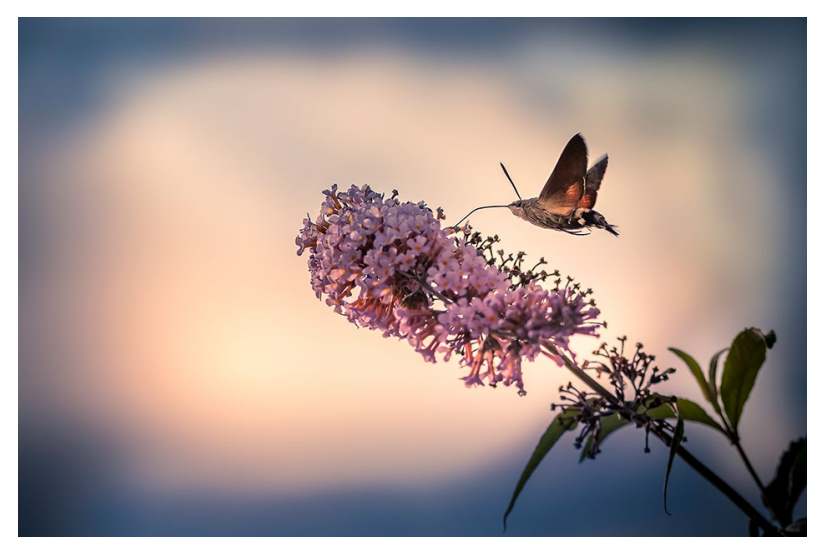

Fig. 1 The hummingbird is often depicted as the animal linking the worlds of the living with those who have already passed away. This butterfly-hummingbird (Macroglossum stellatarum) was surprised in this magic instant by Claudio Ostorero, a passionate lover of nature and a great, but overly modest, photographer. I met him when he was a young man with a reflux nephropathy, more than 20 years ago; chronic kidney disease never kept him away from his passion, the mountain bike, a passion he transfers to the young people he trains. We have shared many moments over time, but it was only recently on Facebook that I discovered his talent. Enjoy his work on Instagram: claudio_osto_photographer (courtesy of the author) 
Funding No funding.

\section{Declarations}

Conflict of interest The authors declares no conflict of interest.

Ethical approval This editorial does not need ethical approval.

\section{Reference}

1. https://www.washingtonpost.com/?utm_campaign=wp_todays_ worldview\&utm_medium $=$ email\&utm_source $=$ newsletter $\&$ wpisrc $=$ nl_todayworld \& carta-url=https $\% 3 \mathrm{~A} \% 2 \mathrm{~F} \% 2 \mathrm{Fs} 2$. washi ngtonpost.com\%2Fcar-ln-tr\%2F1d2150b\%2F6184ac909d2fd a9d4136a520\%2F5e99537aae7e8a0ab1011f7d\%2F5\%2F67\% 2F6184ac909d2fda9d4136a520. Accessed 10 Nov 2021

Publisher's Note Springer Nature remains neutral with regard to jurisdictional claims in published maps and institutional affiliations. 